\title{
COMPUTATION OF THE DELTA IN MULTIDIMENSIONAL JUMP-DIFFUSION SETTING WITH APPLICATIONS TO STOCHASTIC VOLATILITY MODELS
}

\author{
ASMA KHEDHER
}

\begin{abstract}
We study the robustness of option prices to model variation in a multidimensional jump-diffusion framework. In particular we consider price dynamics in which small variations are modeled either by a Poisson random measure with infinite activity or by a Brownian motion. We consider both European and Exotic option and we study their deltas using two approaches: the Malliavin method and the Fourier method. We prove robustness of the deltas to model variation. We apply these results to the study of stochastic volatility models for the underlying and the corresponding options.
\end{abstract}

\section{INTRODUCTION}

In the Black-Scholes option pricing theory, asset prices are modeled by a geometric Brownian motion with a constant volatility parameter. However, it has been observed that the implied volatility depends on the strike price and the expiration date implying the so-called "volatility smile". This shows the limitations of the Black-Scholes model. An alternative is to model the market price processes by jumps and stochastic volatility. These models seem to be more robust and closer to reality. In fact, the market is usually incomplete and one can't hedge alway all the risks (see e.g Cont and Tankov [11] for more about incomplete market).

In this paper, we consider price dynamics driven by multidimensional jump-diffusions. We approximate the small jumps by a multidimensional continuous martingale with appropriately scaled variance. This idea was first initiated by Rydelberg [29] and Asmussen and Rosinski [1] who studied the approximation of small jumps in a Lévy process by a scaled Brownian motion.

We aim to compute the delta of the option written in such models. The delta is defined to be a sensitivity of the option price with respect to the state of the underlying asset. In a complete market, the delta also represents the number of assets to hold in a self financing portfolio replicating the option. It is also important in incomplete market for the construction of partial hedges (see Cont,Tankov, and Voltchkova [12]).

Date: March 29, 2011.

2000 Mathematics Subject Classification. 60H07,60H35, 91G20.

Key words and phrases. delta hedging, Malliavin Calculus, jump-diffusions, stochastic volatility, robustness.

I am thankful to Fred Espen Benth and Giulia Di Nunno for their helpful comments and remarks. 
We consider European and Asian options. In both cases the payoff function is not differentiable. Fournié et al. [21] used a Malliavin approach to derive an expression for the delta not involving any differentiation of the payoff function. This was done for models with deterministic volatility and where the asset price is driven by Brownian motion. In the case of jump-diffusion dynamics where the jump part is driven by a Poisson process, Davis and Johansson [14] propose to use the Malliavin approach only with respect to the Wiener term in the jump-diffusion. Benth, Di Nunno, and Khedher [5] extend this idea to substantially more general jump-diffusion processes. In this paper we extend the approach by Benth, Di Nunno, and Khedher [5] to multidimensional Lévy diffusions and we apply this to the computation of the delta for stochastic volatility models. To compute the delta, we use a Malliavin calculus for jump processes developed by Solé, Utzet, and Vives [32] and Di Nunno [15]. From the point of view of robustness we show that both the price processes and the deltas of the two models converge.

The Asian option has been widely studied. Caramellino and Marchisio [8] and El-Kathib and Privault [18] studied representation formulas for the delta of Asian options using a Malliavin calculus. They considered models in which the jump part is driven by Poisson process. In this paper, we derive an expression for the delta of Asian options written in more general-jump diffusion processes and we prove the robustness of the option price and its delta.

As an application, we consider a general stochastic volatility model. That is we model the price process by a stochastic differential equation in which the volatility $\sigma$ is a function of another process. In that case, $\sigma=f(Y)$, where $f$ is a smooth, positive, and increasing function and $Y$ is a stochastic differential equation driven by a continuous part and a jump part. The continuous part of the process $Y$ is correlated with the Brownian motion of the underlying's price. Cass and Friz [13] compute the delta for stochastic volatility models using the Bismut-Elworthy-Li formula. In this paper, to compute the delta, we consider a Malliavin derivative with respect to the Wiener term of the underlying's price. The weights we obtain involve the stochastic volatility. As an example we consider the Heston model (see Heston [22]) in which the function $f$ is the square root of the process $Y$ and the process $Y$ is a continuous mean-reverting process. We also consider a Heston model which has jumps in the volatility (see Matytsin [25] and Sepp [31]). These models have nice properties, they directly model the observed random behavior of market volatility and allow to reproduce more realistic return distributions, in particular, thicker than lognormal tails. They also provide a closed form solution for European options making it more tractable and easier to implement than other stochastic volatility models.

Moreover, we consider the BN-S model, introduced by Barndorff-Nielson and Shephard [7], in which the stochastic variance of log-returns is constructed via a mean-reverting, stationary process of the Ornstein-Uhlenbeck type driven by a subordinator. That is the variance of the price process is given by

$$
Y(t)=-\lambda Y(t) d t+d Z(t)
$$

where $\lambda>0$ and $Z$ is a subordinator. In applications, the term $\lambda$ is approximated. In this paper, we approximate the term $\lambda$ by $\lambda_{\varepsilon}$ and we investigate the robustness of the model 
and of the associated option price. As the market is incomplete, We consider a structure preserving class of equivalent martingale measures introduced by Nicolato and Venardos $[27]$ and we prove the convergence of the option price after a change of measure in this class. For the computation of the delta of options written in such models, we refer to Benth, Groth, and Wallin [3].

The paper is organized as follows. In section 2 we make a short introduction about Lévy processes. In section 3 we study the computation of the delta and the related analysis of robustness to the model. Section 4 deals with the computation of the delta in stochastic volatility models and the robustness of the BN-S model.

\section{Some MATHEMATICAL PRELIMINARIES}

Let $(\Omega, \mathcal{F}, \mathbb{P})$ be a complete probability space equipped with a filtration $\left\{\mathcal{F}_{t}\right\}_{t \in[0, T]}(T>$ 0 ) satisfying the usual conditions (see Karatzas and Shreve [24]). We introduce the generic notation $L=\left(L^{(1)}(t), \ldots, L^{(d)}(t)\right)^{*}, 0 \leq t \leq T$, for an $\mathbb{R}^{d}$-valued Lévy process on the given probability space and denote by $B=B(t), 0 \leq t \leq T$, a one-dimensional Brownian motion independent of $L$ and $\forall 1 \leq i \leq d, L^{(i)}(0)=B(0)=0$ by convention. Here . denotes $^{*}$ the transpose of a given vector or a given matrix. We work with the RCLL ${ }^{1}$ version of the Lévy process and let $\triangle L(t):=L(t)-L(t-)$. Denote the Lévy measure of $L$ by $\ell(d z)$. The measure $\ell(d z), z \in \mathbb{R}_{0}^{d}$, is a $\sigma$-finite Borel measure on $\mathbb{R}_{0}^{d}:=\mathbb{R}^{d} \backslash\{0\}$. We write

$$
\begin{aligned}
D_{(a, b]} & =\left\{z \in \mathbb{R}^{d} ; \quad a<\|z\| \leq b\right\}, \quad \text { for } \quad 0 \leq a<b<\infty, \\
D_{(a, \infty)} & =\left\{z \in \mathbb{R}^{d} ; \quad a<\|z\|<\infty\right\}, \quad \text { for } \quad 0 \leq a<\infty,
\end{aligned}
$$

where $\|$.$\| is the norm in \mathbb{R}^{d}$. We also recall the Lévy-Itô decomposition of a Lévy process (see Sato [30]):

Theorem 2.1. Let $L$ be a Lévy process on $\mathbb{R}^{d}$ and $\ell$ its Lévy measure. Then we have:

- $\ell=\ell\left(d z_{1}, \ldots, d z_{d}\right)$ verifies

$$
\int_{\mathbb{R}_{0}^{d}} \min \left(1,\|z\|^{2}\right) \ell(d z)<\infty .
$$

- The jump measure of $L$, denoted by $N(d t, d z)=N\left(d t, d z_{1}, \ldots, d z_{d}\right)$, is a Poisson random measure on $[0, \infty) \times \mathbb{R}_{0}^{d}$ with intensity measure $\ell(d z) d t$.

- There exists an $\mathbb{R}^{m}$-Brownian motion $W=\left(W^{(1)}(t), \ldots, W^{(m)}(t)\right)^{*}, 0 \leq t \leq T$, a vector $A \in \mathbb{R}^{d}$, and a symmetric non-negative definite matrix $\Sigma \in \mathcal{L}\left(\mathbb{R}^{m}, \mathbb{R}^{d}\right)$ such that

$$
L(t)=A t+\Sigma W(t)+Z(t)+\lim _{\varepsilon \downarrow 0} \widetilde{Z}_{\varepsilon}(t)
$$

where $Z(t)=\left(Z^{(1)}(t), \ldots, Z^{(d)}(t)\right)^{*}$ such that

$$
Z^{(i)}(t)=\int_{0}^{t} \int_{D(1, \infty)} z_{i} N(d s, d z), \quad \forall \quad 1 \leq i \leq d,
$$

\footnotetext{
${ }^{1}$ Right-continuous with left limits, also called càdlàg.
} 
and the process $\widetilde{Z}_{\varepsilon}(t)=\left(\widetilde{Z}_{\varepsilon}^{(1)}(t), \ldots, \widetilde{Z}_{\varepsilon}^{(d)}(t)\right)^{*}$ such that

$$
\widetilde{Z}_{\varepsilon}^{(i)}(t):=\int_{0}^{t} \int_{D(\varepsilon, 1)} z_{i} \tilde{N}(d s, d z), \quad \forall \quad 1 \leq i \leq d,
$$

Here $\widetilde{N}(d t, d z)$ is a compensated Poisson random measure. The convergence of $\widetilde{Z}_{\varepsilon}(t)$ in (2.1) is almost sure and uniform on $t \in[0, T]$. The components $W, Z$ and $\widetilde{Z}_{\varepsilon}$ are independent.

In the following, we consider that the Poisson random measure takes the form

$$
N(d t, d z)=\left(N^{(1)}(d t, d z), \ldots, N^{(d)}(d t, d z)\right), \quad z \in \mathbb{R}
$$

where $N^{(i)}(d t, d z), 1 \leq i \leq d$, are independent. So that the Lévy measure takes the form $\ell(d z)=\left(\ell_{1}(d z), \ldots, \ell_{d}(d z)\right)$. In that case, the processes $Z^{(i)}(t)$ and $\widetilde{Z}_{\varepsilon}^{(i)}(t)$ are respectively given by

$$
\begin{gathered}
Z^{(i)}(t)=\int_{0}^{t} \int_{|z|>1} z N^{(i)}(d s, d z), \quad \forall \quad 1 \leq i \leq d, \\
\widetilde{Z}^{(i)}(t)=\int_{0}^{t} \int_{\varepsilon<|z| \leq 1} z \widetilde{N}^{(i)}(d s, d z), \quad \forall \quad 1 \leq i \leq d
\end{gathered}
$$

and the Lévy processes $L^{(i)}, 1 \leq i \leq d$, given by (2.1) are independent. The dependent case will be studied in a future work.

In various applications involving statistical and numerical methods, it is often useful to approximate the small jumps by a scaled Brownian motion. This approximation was advocated in Rydberg [29] as a way to simulate the path of a Lévy process with NIG distributed increments and later studied in detail by Asmussen and Rosinski [1]. We shall make use of it to study robustness of option prices and their deltas based on multidimensional jump-diffusion models.

Let $L$ be the d-dimensional Lévy process given by (2.1) with a Poisson random measure given by (2.2). We introduce the following notation for the variation of the Lévy process $L$ close to the origin $\sigma^{2}(\varepsilon)=\left(\sigma_{1}^{2}(\varepsilon), \ldots, \sigma_{d}^{2}(\varepsilon)\right)^{*}$, where

$$
\sigma_{i}^{2}(\varepsilon):=\int_{|z|<\varepsilon} z^{2} \ell_{i}(d z), \quad 0<\varepsilon \leq 1, \quad 1 \leq i \leq d .
$$

Since every Lévy measure $\ell_{i}(d z)$ integrates $z^{2}$ in an open interval around zero, we have that $\sigma_{i}^{2}(\varepsilon), 1 \leq i \leq d$, are finite for any $\varepsilon>0$. Note that the $\sigma_{i}^{2}(\varepsilon)$ is the variance of the jumps of $L^{(i)}$ smaller than $\varepsilon$ in the case $L^{(i)}$ is symmetric and has mean zero. By dominated convergence $\sigma_{i}^{2}(\varepsilon), 1 \leq i \leq d$, converge to zero when $\varepsilon \downarrow 0$.

Recall the Lévy-Itô decomposition of a Lévy process $L$ and introduce now an approximating Lévy process (in law)

$$
L_{\varepsilon}(t):=A t+\Sigma W(t)+\sigma(\varepsilon) B(t)+Z(t)+\widetilde{Z}_{\varepsilon}(t),
$$

with $\sigma^{2}(\varepsilon)$ is as in (2.3) and $B$ is a one-dimensional Brownian motion independent of $L$ (which in particular means independent of $W$ ). From the definition of $\widetilde{Z}_{\varepsilon}^{(i)}, 1 \leq i \leq d$, we 
see that we have substituted the small jumps (compensated by their expectation) in $L^{(i)}$ by a Brownian motion scaled with $\sigma_{i}(\varepsilon)$, the standard deviation of the compensated small jumps. We have the following result taken from Benth, Di Nunno, and Khedher [4].

Proposition 2.2. Let the process $L$, respectively $L_{\varepsilon}$, be defined as in equation (2.1), respectively (2.4). Then, for every $t$,

$$
\lim _{\varepsilon \rightarrow 0} L_{\varepsilon}^{(i)}(t)=L^{(i)}(t) \quad \mathbb{P}-\text { a.s., } \quad \forall \quad 1 \leq i \leq d .
$$

In fact, the limit above also holds in $L^{1}(\Omega, \mathcal{F}, \mathbb{P})$ with

$$
\mathbb{E}\left[\left|L_{\varepsilon}^{(i)}(t)-L^{(i)}(t)\right|\right] \leq 2 \sigma_{i}(\varepsilon) \sqrt{t}, \quad \forall \quad 1 \leq i \leq d .
$$

We shall make use of the approximation and its convergence properties in our analysis.

When we analyze the delta of option prices based on a multidimensional jump-diffusion models, we will use a Malliavin derivative introduced in Solé, Utzet, and Vives [32]. This Malliavin derivative is defined in a subspace of $L^{2}(\Omega)$ and is essentially a derivative with respect to the Brownian part of $L$. We will denote it by $D_{t, 0}$. Its dual, the Skorohod integral is also defined by Solé, Utzet and Vives [32]. We will denote it by $\delta$. For the Malliavin calculus with respect to Lévy processes we have to refer to Di Nunno, Øksendal, and Proske [16], Di Nunno [15], and Di Nunno and Rozanov [17].

\section{RoBUstness OF OPTION PRICES AND THEIR DELTAS}

3.1. Robustness of option prices. In this section we consider the robustness of jumpdiffusions given by the solution of stochastic differential equations of the form $X(t)=$ $\left(X^{(1)}(t), \ldots, X^{(d)}(t)\right)$, where

$$
\begin{aligned}
X^{(i)}(t) & =x_{i}+\int_{0}^{t} \alpha_{i}(X(s-)) d s+\int_{0}^{t} \sum_{j=1}^{m} \beta_{i j}(X(s-)) d W^{(j)}(s) \\
& +\int_{0}^{t} \int_{\mathbb{R}_{0}} \gamma_{i}(X(s-), z) \widetilde{N}^{(i)}(d s, d z), \quad 1 \leq i \leq d .
\end{aligned}
$$

Here $x_{i} \in \mathbb{R}, \alpha_{i}, \beta_{i j}$ are measurable functions $\mathbb{R}^{d} \longrightarrow \mathbb{R}$, and $\gamma_{i}$ is a measurable function $\mathbb{R}^{d} \times \mathbb{R}_{0} \longrightarrow \mathbb{R}$. We assume, moreover, that the coefficient functions $\alpha_{i}(x)$ and $\beta_{i j}(x)$ have linear growth and are Lipschitz continuous. Each $\gamma_{i}(x, z)$ is of the form $\gamma_{i}(x, z)=\delta_{i}(x) g_{i}(z)$, where the (stochastic) factor $\delta_{i}(x)$ has linear growth and is Lipschitz continuous and the (deterministic) factors $g_{i}(z)$ satisfy

$$
G^{2}(\infty)=\sum_{i=1}^{d} \int_{\mathbb{R}_{0}} g_{i}^{2}(z) \ell_{i}(d z)<\infty,
$$

which will ensure that $\forall 1 \leq i \leq d, X^{(i)}(t)$ has finite variance. We also define

$$
G_{i}^{2}(\varepsilon)=\int_{|z|<\varepsilon} g_{i}^{2}(z) \ell_{i}(d z), \quad 1 \leq i \leq d
$$


and

$$
G^{2}(\varepsilon)=\sum_{i=1}^{d} \int_{|z|<\varepsilon} g_{i}^{2}(z) \ell_{i}(d z),
$$

for later use.

Note that we consider a stochastic differential equation with the roles of $W$ and $\tilde{N}$ separated, that is, we do not consider an equation using $L$ as the integrator, but rather split the roles of the continuous martingale and the pure-jump parts. This is more in line with common formulations of such stochastic differential equations (see for example Davis and Johansson [14]). Introduce the approximating jump-diffusion dynamics where the small jumps part in (3.1) has been substituted by the Brownian motion B independent of $\mathrm{W}$ and appropriately scaled, namely $X_{\varepsilon}(t)=\left(X_{\varepsilon}^{(1)}(t), \ldots, X_{\varepsilon}^{(d)}(t)\right)$, where

$$
\begin{aligned}
X_{\varepsilon}^{(i)}(t) & =x_{i}+\int_{0}^{t} \alpha_{i}\left(X_{\varepsilon}(s-)\right) d s+\int_{0}^{t} \sum_{j=1}^{m} \beta_{i j}\left(X_{\varepsilon}(s-)\right) d W^{(j)}(s) \\
& +\int_{0}^{t}\left(\int_{|z|<\varepsilon}\left(\gamma_{i}^{2}\left(X_{\varepsilon}(s-), z\right) \ell_{i}(d z)\right)^{\frac{1}{2}} d B(s)+\int_{0}^{t} \int_{|z| \geq \varepsilon} \gamma_{i}\left(X_{\varepsilon}(s-), z\right) \tilde{N}^{(i)}(d s, d z)\right. \\
& =x_{i}+\int_{0}^{t} \alpha_{i}\left(X_{\varepsilon}(s-)\right) d s+\int_{0}^{t} \sum_{j=1}^{m} \beta_{i j}\left(X_{\varepsilon}(s-)\right) d W^{(j)}(s) \\
& +\int_{0}^{t} G_{i}(\varepsilon) \delta_{i}\left(X_{\varepsilon}(s-)\right) d B(s)+\int_{0}^{t} \int_{|z| \geq \varepsilon} \gamma_{i}\left(X_{\varepsilon}(s-), z\right) \tilde{N}^{(i)}(d s, d z) .
\end{aligned}
$$

The existence and uniqueness of the solutions $X(t)$ and $X_{\varepsilon}(t)$ are ensured by the following theorem collected from Ikeda and Watanabe [23] (Thm 9.1. Chap IV):

Theorem 3.1. Let $U$ be an open set in $\mathbb{R}_{0}$, $\alpha$ be a measurable function $\mathbb{R}^{d} \longrightarrow \mathbb{R}^{d}, \beta$ be a measurable function $\mathbb{R}^{d} \longrightarrow \mathbb{R}^{d} \times \mathbb{R}^{m}$, and $\gamma$ be a measurable function $\mathbb{R}^{d} \times U \longrightarrow \mathbb{R}^{d}$ such that, for some positive constant $K$,

$$
\|\alpha(x)\|^{2}+\|\beta(x)\|^{2}+\int_{U}\|\gamma(x, z)\|^{2} \ell(d z) \leq K\left(1+\|x\|^{2}\right), \quad x \in \mathbb{R}^{d}
$$

$\|\alpha(x)-\alpha(y)\|^{2}+\|\beta(x)-\beta(y)\|^{2}+\int_{U}\|\gamma(x, z)-\gamma(y, z)\|^{2} \ell(d z) \leq K\|x-y\|^{2}, \quad x, y \in \mathbb{R}^{d}$.

Then there exists a unique d-dimensional $\mathcal{F}_{t}$-adapted right-continuous process $X(t)$ with left-hand limits which satisfies the following stochastic differential equation

$$
\begin{aligned}
X^{(i)}(t)=x_{i}+\int_{0}^{t} \alpha_{i}(X(s-)) d s+\int_{0}^{t} \sum_{j=1}^{m} \beta_{i j}(X(s-)) d W^{(j)}(s) \\
+\int_{0}^{t} \int_{U} \gamma_{i}(X(s-), z) \tilde{N}^{(i)}(d s, d z), \quad 1 \leq i \leq d .
\end{aligned}
$$


In Prop.3.3 in Benth, Di Nunno, and Khedher [5], we prove the convergence of $X_{\varepsilon}(t)$ to $X(t)$, where $X(t)$ is a one-dimensional stochastic differential equation. In the same way, we prove the following result

Proposition 3.2. For every $0 \leq t \leq T<\infty$, we have

$$
\sum_{i=1}^{d}\left\|X^{(i)}(t)-X_{\varepsilon}^{(i)}(t)\right\|_{2}^{2} \leq C G^{2}(\varepsilon), \quad \forall \quad 1 \leq i \leq d,
$$

where $X^{(i)}$ and $X_{\varepsilon}^{(i)}$ are solutions of (3.1) and (3.2), respectively and $C$, is a positive constant depending on $T$, but independent of $\varepsilon$.

From Proposition 3.2, we can deduce the following result.

Proposition 3.3. Let $X^{(i)}$ and $X_{\varepsilon}^{(i)}$ be solutions of (3.1) and (3.2), respectively. For every $0 \leq t \leq T<\infty$, we have

$$
\sum_{i=1}^{d}\left\|\int_{0}^{T}\left\{X^{(i)}(t)-X_{\varepsilon}^{(i)}(t)\right\} d t\right\|_{2}^{2} \leq C^{\prime} G^{2}(\varepsilon), \quad \forall \quad 1 \leq i \leq d,
$$

where $C^{\prime}$ is a positive constant depending on $T$, but independent of $\varepsilon$.

Proof. By Hölder inequality and Proposition 3.2, $\forall 1 \leq i \leq d$, we have

$$
\begin{aligned}
\sum_{i=1}^{d}\left\|\int_{0}^{T}\left\{X^{(i)}(t)-X_{\varepsilon}^{(i)}(t)\right\} d t\right\|_{2}^{2} & \leq \sum_{i=1}^{d} T \mathbb{E}\left[\int_{0}^{T}\left\{X^{(i)}(t)-X_{\varepsilon}^{(i)}(t)\right\}^{2} d t\right] \\
& \leq \sum_{i=1}^{d} T \int_{0}^{T} \mathbb{E}\left[\left\{X^{(i)}(t)-X_{\varepsilon}^{(i)}(t)\right\}^{2}\right] d t \\
& \leq T^{2} C G^{2}(\varepsilon)
\end{aligned}
$$

and the result follows.

Moreover, we have the following robustness of option prices.

Corollary 3.4. Suppose $f: \mathbb{R}^{d} \longrightarrow \mathbb{R}$ is a Lipschitz continuous function and $X$ and $X_{\varepsilon}$ solve (3.1) and (3.2), resp. Then, for every $0 \leq t \leq T<\infty$, there exists two positive constants $C$ and $C^{\prime}$ depending on $T$ but independent of $\varepsilon$ such that

$$
\left|\mathbb{E}\left[f\left(X_{\varepsilon}(t)\right)\right]-\mathbb{E}[f(X(t))]\right| \leq C G(\varepsilon)
$$

and

$$
\left|\mathbb{E}\left[f\left(\int_{0}^{T} X_{\varepsilon}(t) d t\right)\right]-\mathbb{E}\left[f\left(\int_{0}^{T} X(t) d t\right)\right]\right| \leq C^{\prime} G(\varepsilon) .
$$

Proof. Letting $K$ be the Lipschitz constant of $f$, we have from the Jensen inequality,

$$
\left|\mathbb{E}\left[f\left(X_{\varepsilon}(t)\right)\right]-\mathbb{E}[f(X(t))]\right| \leq K \mathbb{E}\left[\left\|X_{\varepsilon}(t)-X(t)\right\|\right]
$$




$$
\leq K\left(\sum_{i=1}^{d} \mathbb{E}\left[\left|X_{\varepsilon}^{(i)}(t)-X^{(i)}(t)\right|^{2}\right]\right)^{\frac{1}{2}} .
$$

The latter follows from the Cauchy-Schwarz inequality. Applying Prop. 3.2, the result follows. Moreover, we have

$$
\left|\mathbb{E}\left[f\left(\int_{0}^{T} X_{\varepsilon}(t) d t\right)\right]-\mathbb{E}\left[f\left(\int_{0}^{T} X(t) d t\right)\right]\right| \leq K \mathbb{E}\left[\left\|\int_{0}^{T}\left\{X_{\varepsilon}(t)-X(t)\right\} d t\right\|\right]
$$

Hence, from the Cauchy-Schwarz inequality and Prop. 3.3, the result follows.

3.2. Computation of the Delta and robustness. In this section we present the Malliavin approach to compute the delta for option prices based on a jump-diffusion market model. We consider an approach studied in Benth, Di Nunno, and Khedher [5] which is based on a separability assumption. We assume that the diffusion matrix $\beta \in \mathcal{L}\left(\mathbb{R}^{d}, \mathbb{R}^{d}\right)$ has an inverse $\beta^{-1}$ and satisfies the uniform ellipticity condition

$$
\exists \eta>0 ; \quad \xi^{*} \beta^{*}(x) \beta(x) \xi \geq \eta|\xi|^{2}, \quad \text { for any } \xi, x \in \mathbb{R}^{d} .
$$

Separability approach. Let $\mathcal{F}_{t}^{\widetilde{N}}=\sigma\left\{\int_{0}^{s} \int_{U}\left(\widetilde{N}^{(1)}(d u, d z), \ldots, \widetilde{N}^{(d)}(d u, d z)\right) ; \quad s \leq t, \quad U \in\right.$ $\left.\mathcal{B}\left(\mathbb{R}_{0}\right)\right\}$. Assume that $\forall 1 \leq i \leq d, \alpha_{i}, \beta_{i}$, and $\gamma_{i}$ are continuously differentiable functions with bounded derivatives and consider Markov jump diffusions, $X^{(i)}$ of the form (3.1), for which we have a continuously differentiable functions $h_{i}: \mathbb{R}^{2} \longrightarrow \mathbb{R}$ with bounded derivative in the first argument such that

$$
X^{(i)}(t)=h_{i}\left(X^{c(i)}(t), X^{J(i)}(t)\right), \quad X^{(i)}(0)=x_{i}, \quad 1 \leq i \leq d .
$$

Here $X^{c(i)}$ satisfies a stochastic differential equation

$$
\begin{aligned}
d X^{c(i)}(t) & =\alpha_{c i}\left(X^{c(i)}(t)\right) d t+\sum_{j=1}^{d} \beta_{c i j}\left(X^{c(i)}(t)\right) d W^{(j)}(t) \\
X^{c(i)}(0) & =x_{i}=h_{i}\left(X^{c(i)}(0), X^{J(i)}(0)\right)
\end{aligned}
$$

with continuously differentiable coefficients $\alpha_{c i}, \beta_{c i j}$, while $X^{J(i)}$ is adapted to the natural filtration $\mathcal{F}^{\widetilde{N}}$ of the compensated compound Poisson process $\widetilde{N}$. In particular, $X^{J(i)}$ does not depend on $x_{i}$. The jump-diffusion process of type (3.8) is called separable.

We associate to the process $X^{c}$, a process $V$ given by

$$
V(t)=I+\int_{0}^{t} \alpha_{c}^{\prime}\left(X^{c}(s)\right) V(s) d s+\int_{0}^{t} \sum_{i=1}^{d} \beta_{c i}^{\prime}\left(X^{c}(s)\right) V(s) d W^{(i)}(s),
$$

where $I$ is the identity matrix, $\alpha_{c}=\left(\alpha_{c(1)}, \ldots, \alpha_{c(d)}\right)^{*}, \beta_{c i}$ is the i-th column vector of $\beta_{c}$, and prime denotes derivatives. The process $V$ is called the first variation process for $X^{c}$ and we have

$$
V(t)=\nabla X^{c}(t)
$$


We provide an example of a jump-diffusion dynamics satisfying our assumptions. Consider a jump-diffusion of the form

$$
\begin{aligned}
d X^{(1)}(t)= & \alpha_{1}\left(X^{(2)}(t), \ldots, X^{(d)}(t)\right) X^{(1)}\left(t^{-}\right) d t+\beta_{1}\left(X^{(2)}(t), \ldots, X^{(d)}(t)\right) X^{(1)}\left(t^{-}\right) d W(t) \\
+ & \int_{\mathbb{R}_{0}}\left(e^{z}-1\right) X^{(1)}(t-) \tilde{N}(d t, d z), \quad X^{(1)}(0)=x_{1}, \\
d X^{(i)}(t)= & \alpha_{i}\left(X^{(2)}(t), \ldots, X^{(d)}(t)\right) d t+\beta_{i}\left(X^{(2)}(t), \ldots, X^{(d)}(t)\right)\left(t^{-}\right) d W(t), \\
& X^{(i)}(0)=x_{i}, \quad i=2, \ldots, d,
\end{aligned}
$$

where $\alpha_{i}$ and $\beta_{i}$ are constants. We introduce the process $X^{c(1)}(t)$ defined by

$$
\begin{aligned}
d X^{c(1)}(t)= & \left(\alpha_{1}\left(X^{c(2)}(t), \ldots, X^{c(d)}(t)\right)+\int_{\mathbb{R}_{0}}\left(1+z-e^{z}\right) \ell(d z)\right) X^{c(1)}(t) d t \\
+ & \beta_{1}\left(X^{c(2)}(t), \ldots, X^{c(d)}(t)\right) X^{c(1)}(t) d W(t), \quad X^{c(1)}(0)=x_{1}, \\
d X^{c(i)}(t)= & \alpha_{i}\left(X^{c(2)}(t), \ldots, X^{c(d)}(t)\right) X^{c(1)}(t) d t+\beta_{i}\left(X^{c(2)}(t), \ldots, X^{c(d)}(t)\right) X^{c(1)}(t) d W(t), \\
& X^{c(i)}(0)=x_{i}, \quad i=2, \ldots, d,
\end{aligned}
$$

Then by applying the Itô formula to

$$
\begin{aligned}
\widehat{X}^{(1)}(t) & =e^{\widetilde{Z}(t)} X^{c(1)}(t), \quad \widetilde{Z}(t)=\int_{\mathbb{R}_{0}} z \widetilde{N}(d t, d z), \\
\widehat{X}^{(i)}(t) & =X^{c(i)}(t),
\end{aligned}
$$

we can prove that $\widehat{X}(t)=X(t) \quad$ a.e.

We define the payoff function $f=f\left(X\left(t_{1}\right), \ldots, X\left(t_{n}\right)\right)$ to be a square integrable function discounted from maturity $T$ and evaluated at the times $t_{1}, \ldots, t_{n}$. We are interested on differentiating with respect to the state of the underlying asset expectations of the form

$$
v(x)=\mathbb{E}\left[f\left(X\left(t_{1}\right), \ldots, X\left(t_{n}\right)\right)\right] .
$$

The following result is the extension of the Theorem 4.1 in Benth, Di Nunno, and Khedher [5] for the computation of the delta for a European option written in a multidimensional jump-diffusion.

Theorem 3.5. Let $X$ be a diffusion of the form (3.1). We assume the uniform ellipticity condition (3.7) and the separability condition. Define

$$
\Gamma=\left\{a \in L^{2}[0, T] \mid \int_{0}^{t_{i}} a(t) d t=1, \quad \forall i=1, \ldots, n\right\} .
$$

Then for $a \in \Gamma$ and $f\left(X\left(t_{1}\right), \ldots, X\left(t_{n}\right)\right)$ square integrable, we have

$$
\Delta=(\nabla v(x))^{*}=\mathbb{E}\left[f\left(X\left(t_{1}\right), \ldots, X\left(t_{n}\right)\right) \int_{0}^{T} a(t)\left(\beta_{c}^{-1}\left(X^{c}(t)\right) V(t)\right)^{*} d W(t)\right],
$$

where $V$ is given by (3.10). 
Proof. Assume that $f \in C_{K}^{\infty}\left(\mathbb{R}^{d} \times \ldots \times \mathbb{R}^{d}\right)$, the set of infinitely differentiable functions $\mathbb{R}^{d} \times \ldots \times \mathbb{R}^{d} \longrightarrow \mathbb{R}$ with compact support. We denote by $\nabla_{i} f\left(X\left(t_{1}\right), \ldots, X\left(t_{n}\right)\right)$ the gradient of $f$ with respect to $X\left(t_{i}\right)$ and by $\frac{\partial X\left(t_{i}\right)}{\partial x}$ the $d \times d$ matrix of derivatives of the $d$-dimensional random variable $X\left(t_{i}\right)$ with respect to its initial condition. Then

$$
\begin{aligned}
\nabla v(x) & =\mathbb{E}\left[\sum_{i=1}^{n} \nabla_{i} f\left(X\left(t_{1}\right), \ldots, X\left(t_{n}\right)\right) \frac{\partial X\left(t_{i}\right)}{\partial x}\right] \\
& =\mathbb{E}\left[\sum_{i=1}^{n} \nabla_{i} f\left(X\left(t_{1}\right), \ldots, X\left(t_{n}\right)\right) \frac{\partial X\left(t_{i}\right)}{\partial X^{c}\left(t_{i}\right)} V\left(t_{i}\right)\right],
\end{aligned}
$$

where $V$ is the first variation process for $X^{c}$. By the chain rule (Corollary 3.6 in Solé, Utzet and Vives [32]), we have

$$
D_{t, 0} X\left(t_{i}\right)=\frac{\partial X\left(t_{i}\right)}{\partial X^{c}\left(t_{i}\right)} D_{t}^{W} X^{c}\left(t_{i}\right)=\frac{\partial X\left(t_{i}\right)}{\partial X^{c}\left(t_{i}\right)} V\left(t_{i}\right)(V(t))^{-1} \beta_{c}\left(X^{c}(t)\right) \mathbf{1}_{\left\{t \leq t_{i}\right\}},
$$

where $D^{W}$ is the Malliavin derivative with respect to the Brownian motion $W$. Therefore,

$$
\frac{\partial X\left(t_{i}\right)}{\partial X^{c}\left(t_{i}\right)} V\left(t_{i}\right) \mathbf{1}_{\left\{t \leq t_{i}\right\}}=D_{t, 0} X\left(t_{i}\right) V(t) \beta_{c}^{-1}\left(X^{c}(t)\right) .
$$

Multiply by $a(t) \in \Gamma$ and integrate,

$$
\frac{\partial X\left(t_{i}\right)}{\partial X^{c}\left(t_{i}\right)} V\left(t_{i}\right)=\int_{0}^{T} D_{t, 0} X\left(t_{i}\right) a(t) \beta_{c}^{-1}\left(X^{c}(t)\right) V(t) d t .
$$

Inserting (3.12) in (3.11), the chain rule (Corollary 3.6 in Solé, Utzet and Vives [32]) yields

$$
\begin{aligned}
\nabla v(x) & =\mathbb{E}\left[\int_{0}^{T} \sum_{i=1}^{n} \nabla_{i} f\left(X\left(t_{1}\right), \ldots, X\left(t_{n}\right)\right) D_{t, 0} X\left(t_{i}\right) a(t) \beta_{c}^{-1}\left(X^{c}(t)\right) V(t) d t\right] \\
& =\mathbb{E}\left[\int_{0}^{T} D_{t, 0} f\left(X\left(t_{1}\right), \ldots, X\left(t_{n}\right)\right) a(t) \beta_{c}^{-1}\left(X^{c}(t)\right) V(t) d t\right] .
\end{aligned}
$$

Since the diffusion matrix $\beta_{c}$ is elliptic, we deduce that $a(t) \beta_{c}^{-1}\left(X^{c}(t)\right) V(t) \in L^{2}(\Omega \times[0, T])$. Therefore using the Duality formula (see Section 6 in Solé, Utzet and Vives [32]), we get

$$
(\nabla v(x))^{*}=\mathbb{E}\left[f\left(X\left(t_{1}, \ldots, X\left(t_{n}\right)\right) \int_{0}^{T} a(t)\left(\beta_{c}^{-1}\left(X^{c}(t)\right) V(t)\right)^{*} d W(t)\right] .\right.
$$

We can extend this formula to square integrable functions of the form $f\left(X\left(t_{1}\right), \ldots, X\left(t_{n}\right)\right)$ following the Proposition A.2 in the Appendix in Benth, Di Nunno, and Khedher [5].

Now we consider the case of an Asian option with payoff of the form $f\left(\int_{0}^{T} X(t) d t\right)$. In the following theorem we give the formula for the derivative with respect to the initial condition in dimension one. 
Theorem 3.6. Let $X$ be a diffusion of the form (3.1) with $d=1$. Let $f(\omega)=f(Z(\omega))$, where $Z(T)=\int_{0}^{T} X(t) d t$. We assume the uniform ellipticity condition (3.7) and the separability condition. Then for $f(Z(\omega)) \in L^{2}(\Omega)$,

$$
\Delta=\mathbb{E}\left[f\left(\int_{0}^{T} X(t) d t\right) \delta\left(2 V^{2}(t) \frac{\partial X(t)}{\partial X^{c}(t)}\left\{\beta_{c}\left(X^{c}(t)\right) \int_{0}^{T} \frac{\partial X(u)}{\partial X^{c}(u)} V(u) d u\right\}^{-1}\right)\right],
$$

where $V$ is given by (3.10).

Proof. Assume that $f \in C_{K}^{\infty}(\mathbb{R})$. Then

$$
\begin{aligned}
\frac{\partial}{\partial x} \mathbb{E}\left[f\left(\int_{0}^{T} X(t) d t\right)\right] & =\mathbb{E}\left[f^{\prime}\left(\int_{0}^{T} X(t) d t\right) \int_{0}^{T} \frac{\partial X(t)}{\partial x} d t\right] \\
& =\mathbb{E}\left[f^{\prime}\left(\int_{0}^{T} X(t) d t\right) \int_{0}^{T} \frac{\partial X(t)}{\partial X^{c}(t)} V(t) d t\right],
\end{aligned}
$$

where $V$ is the first variation process for $X^{c}$. Consider a random variable $\eta \in L^{2}(\Omega \times[0, T])$. Then by the chain rule (Corollary 3.6 in Solé, Utzet and Vives [32]), we have

$$
\begin{aligned}
& \left.\mathbb{E}\left[\int_{0}^{T} D_{u, 0} f\left(\int_{0}^{T} X(t)\right) d t\right) \eta(u) d u\right] \\
= & \mathbb{E}\left[\int_{0}^{T}\left\{f^{\prime}\left(\int_{0}^{T} X(t) d t\right) D_{u, 0}\left(\int_{0}^{T} X(t) d t\right) \eta(u)\right\} d u\right] \\
= & \mathbb{E}\left[f^{\prime}\left(\int_{0}^{T} X(t) d t\right) \int_{0}^{T}\left(\int_{0}^{T} D_{u, 0} X(t) d t\right) \eta(u) d u\right] \\
= & \mathbb{E}\left[f^{\prime}\left(\int_{0}^{T} X(t) d t\right) \int_{0}^{T} \eta(u)\left(\int_{u}^{T} \frac{\partial X(t)}{\partial X^{c}(t)} V(t) V^{-1}(u) \beta_{c}\left(X^{c}(u)\right) d t\right) d u\right] \\
= & \mathbb{E}\left[f^{\prime}\left(\int_{0}^{T} X(t) d t\right) \int_{0}^{T}\left(\int_{0}^{t} \eta(u) V^{-1}(u) \beta_{c}\left(X^{c}(u)\right) d u\right) \frac{\partial X(t)}{\partial X^{c}(t)} V(t) d t\right]
\end{aligned}
$$

We choose

$$
\eta(u)=2 V^{2}(u) \frac{\partial X(u)}{\partial X^{c}(u)} \beta_{c}^{-1}\left(X^{c}(u)\right)\left(\int_{0}^{T} \frac{\partial X(t)}{\partial X^{c}(t)} V(t) d t\right)^{-1} .
$$

Using the fact that $2 \int_{0}^{T} \int_{0}^{t} f(u) f(v) d u d v=\left(\int_{0}^{T} f(s) d s\right)^{2}$, we get

$$
\begin{aligned}
& \mathbb{E}\left[f^{\prime}\left(\int_{0}^{T} X(t) d t\right) \int_{0}^{T}\left(\int_{0}^{t} \eta(u) V^{-1}(u) \beta_{c}\left(X^{c}(u)\right) d u\right) \frac{\partial X(t)}{\partial X^{c}(t)} V(t) d t\right] \\
= & \mathbb{E}\left[f^{\prime}\left(\int_{0}^{T} X(t) d t\right) \int_{0}^{T} \frac{\partial X(t)}{\partial X^{c}(t)} V(t) d t\right] .
\end{aligned}
$$

The result, for $f \in C_{K}^{\infty}(\mathbb{R})$, follows from the duality formula (Section 6 in Solé, Utzet and Vives [32]). We can extend this formula to $f(Z(w)) \in L^{2}(\Omega)$ following the Proposition A.2 in the Appendix in Benth, Di Nunno, and Khedher [5]. 
We next address the question of robustness of the delta with respect to approximations of the small jumps by an appropriately scaled continuous martingale. It turns out that this question can be efficiently answered by means of Fourier transform. The methods of Fourier transform will translate the question of convergence of the delta to a question of convergence of the derivative of the characteristic function of the approximating dynamics. One may ask why we do not study the expression derived above for the delta directly. The reason is that in the singular case of $\beta=0$, the expressions inside the expectation for the delta in Thm 3.5 will involve singular weights which in general are hard to study in the limit (see Benth, Di Nunno, and Khedher [4] for simple examples of such singular weights). The Fourier approach avoids this problem.

The approach we choose can be used also for efficient computations of the delta, however, only for those cases where the characteristic function is easily computable which is in general not the case for stochastic differential equations like (3.1) and (3.2). We also note that the application of the Fourier transform requires also the explicit solution of the first variation process dynamics (3.18).

Assume that $f \in L^{1}\left(\mathbb{R}^{d}\right)$, the space of integrable functions on $\mathbb{R}^{d}$. The Fourier transform of $f$ is defined by

$$
\widehat{f}(u)=\int_{\mathbb{R}^{d}} f(y) e^{\mathrm{i} u \cdot y} d y
$$

where $u$ and $y$ are two d-dimensional vectors and $u \cdot y$ is the standard scalar product in $\mathbb{R}^{d}$. Suppose in addition that $\widehat{f} \in L^{1}\left(\mathbb{R}^{d}\right)$. Then the inverse Fourier transform is well-defined, and we have

$$
f(y)=\frac{1}{(2 \pi)^{d}} \int_{\mathbb{R}^{d}} e^{-\mathrm{i} u \cdot y} \widehat{f}(u) d u .
$$

We refer to Folland [20] for definitions and results on the Fourier transform. Following Carr and Madan [9], we calculate,

$$
\begin{aligned}
\mathbb{E}\left[f\left(X_{\varepsilon}^{x}(t)\right)\right] & =\int_{\mathbb{R}^{d}}\left\{\frac{1}{(2 \pi)^{d}} \int_{\mathbb{R}^{d}} e^{-i y \cdot u} \widehat{f}(u) d u\right\} P_{X_{\varepsilon}^{x}(t)}(d y) \\
& =\frac{1}{(2 \pi)^{d}} \int_{\mathbb{R}^{d}}\left\{\int_{\mathbb{R}^{d}} e^{-i u \cdot y} P_{X_{\varepsilon}^{x}(t)}(d y)\right\} \widehat{f}(u) d u \\
& =\frac{1}{(2 \pi)^{d}} \int_{\mathbb{R}^{d}} \widehat{f}(u) \mathbb{E}\left[\mathrm{e}^{-\mathrm{i} u \cdot X_{\varepsilon}^{x}(t)}\right] d u,
\end{aligned}
$$

where $P_{X_{\varepsilon}^{x}(t)}(d y)$ is the distribution of $X_{\varepsilon}(t)=X_{\varepsilon}^{x}(t)$, the solution of $(3.2)$ with $X_{\varepsilon}(0)=$ $X_{\varepsilon}^{x}(0)=x$. Fubini-Tonelli's Theorem (see Folland [20]) is applied to commute the integrations. Similarily, we get for $X(t)=X^{x}(t)$ being the solution of $(3.1)$ with $X(0)=X^{x}(0)=$ $x$

$$
\mathbb{E}\left[f\left(X^{x}(t)\right)\right]=\frac{1}{(2 \pi)^{d}} \int_{\mathbb{R}^{d}} \widehat{f}(u) \mathbb{E}\left[\mathrm{e}^{-\mathrm{i} u \cdot X^{x}(t)}\right] d u
$$


Thus, in order to study the delta, we need to be able to move differentiation inside the inverse Fourier transform. But, furthermore, we must have accessible the derivative of $X_{\varepsilon}^{x}(t)$ and $X^{x}(t)$ with respect to $x$. Before moving on with the robustness of deltas, we study this.

Introduce $Y(t)=\left(Y^{(i, j)}(t)\right)_{i=1, \ldots, d, j=1, \ldots, d}=\left(\frac{\partial X^{(i)}(t)}{\partial x_{j}}\right)_{i=1, \ldots, d, j=1, \ldots, d}$, where each $Y^{(i, j)}$ satisfies the following stochastic differential equation

$$
\begin{aligned}
Y^{(i, j)}(t)=\sigma & +\int_{0}^{t} \sum_{k=1}^{d} \partial_{k} \alpha_{i}\left(X^{x}(s-)\right) Y^{(k, j)}(s-) d s \\
& +\int_{0}^{t} \sum_{k=1}^{d} \sum_{n=1}^{d} \partial_{k} \beta_{i n}\left(X^{x}(s-)\right) Y^{(k, j)}(s-) d W^{(n)}(s) \\
& +\int_{0}^{t} \int_{\mathbb{R}_{0}} \sum_{k=1}^{d} \partial \gamma_{i}\left(X^{x}(s-), z\right) Y^{(k, j)}(s-) \widetilde{N}^{(i)}(d s, d z),
\end{aligned}
$$

where $\sigma=1$ if $i=j$ and $\sigma=0$ if $i \neq j$. Since the derivatives of $\alpha_{i}, \beta_{i k}$ and $\gamma_{i}$ are assumed to be bounded, it follows from Thm. 3.1 that there exists a unique solution $Y(t)$ of (3.18). From Thm 40 in Chapter $\mathrm{V}$ of Protter [28], it follows that $X^{x}(t)$ is differentiable with respect to $x$, and that

$$
\nabla X^{x}(t)=Y(t)
$$

By the same considerations, $X_{\varepsilon}^{x}(t)$ is differentiable with respect to $x$, and

$$
\nabla X_{\varepsilon}^{x}(t)=Y_{\varepsilon}(t),
$$

with $Y_{\varepsilon}(t)=\left(Y_{\varepsilon}^{(i, j)}(t)\right)_{i=1, \ldots, d, j=1, \ldots, d}=\left(\frac{\partial X_{\varepsilon}^{(i)}(t)}{\partial x_{j}}\right)_{i=1, \ldots, d, j=1, \ldots, d}$, where each $Y_{\varepsilon}^{(i, j)}$ satisfies the following stochastic differential equation

$$
\begin{aligned}
Y_{\varepsilon}^{(i, j)}(t)=\sigma & +\int_{0}^{t} \sum_{k=1}^{d} \partial_{k} \alpha_{i}\left(X_{\varepsilon}^{x}(s-)\right) Y_{\varepsilon}^{(k, j)}(s-) d s \\
& +\int_{0}^{t} \sum_{k=1}^{d} \sum_{n=1}^{d} \partial_{k} \beta_{i n}\left(X_{\varepsilon}^{x}(s-)\right) Y_{\varepsilon}^{(k, j)}(s-) d W^{(n)}(s) \\
& +\int_{0}^{t} G_{i}(\varepsilon) \sum_{k=1}^{d} \partial_{k} \delta_{i}\left(X_{\varepsilon}^{x}(s-)\right) Y_{\varepsilon}^{(k, j)}(s-) d B(s) \\
& +\int_{0}^{t} \int_{|z| \geq \varepsilon} \sum_{k=1}^{d} \partial_{k} \gamma_{i}\left(X_{\varepsilon}^{x}(s-), z\right) Y_{\varepsilon}^{(k, j)}(s-) \widetilde{N}^{(i)}(d s, d z) .
\end{aligned}
$$

In the next Proposition we derive the expressions for the delta based on $X$ and $X_{\varepsilon}$ using the Fourier method. 
Proposition 3.7. Let $X^{x}(t)$ and $Y^{y}(t)$ be solutions of (3.1) and (3.18), resp., and $X_{\varepsilon}^{x}(t)$ and $Y_{\varepsilon}^{y}(t)$ of (3.2) and (3.22), resp. Let $u \widehat{f}(u) \in L^{1}\left(\mathbb{R}^{d}\right)$. Then, for $0 \leq t \leq T$,

$$
\begin{aligned}
\nabla \mathbb{E}\left[f\left(X^{x}(t)\right)\right] & =\frac{1}{(2 \pi)^{d}} \int_{\mathbb{R}^{d}} \widehat{f}(u) \mathbb{E}\left[-\mathrm{i} u Y(t) \mathrm{e}^{-\mathrm{i} u \cdot X^{x}(t)}\right] d u \\
\nabla \mathbb{E}\left[f\left(X_{\varepsilon}^{x}(t)\right)\right] & =\frac{1}{(2 \pi)^{d}} \int_{\mathbb{R}^{d}} \widehat{f}(u) \mathbb{E}\left[-\mathrm{i} u Y_{\varepsilon}(t) \mathrm{e}^{-\mathrm{i} u \cdot X_{\varepsilon}^{x}(t)}\right] d u \\
\nabla \mathbb{E}\left[f\left(\int_{0}^{T} X^{x}(t) d t\right)\right] & =\frac{1}{(2 \pi)^{d}} \int_{\mathbb{R}^{d}} \widehat{f}(u) \mathbb{E}\left[\left\{\int_{0}^{T}-\mathrm{i} u Y(t) d t\right\} \mathrm{e}^{-\mathrm{i} u \cdot \int_{0}^{T} X^{x}(t) d t}\right] d u \\
\nabla \mathbb{E}\left[f\left(\int_{0}^{T} X_{\varepsilon}^{x}(t) d t\right)\right] & =\frac{1}{(2 \pi)^{d}} \int_{\mathbb{R}^{d}} \widehat{f}(u) \mathbb{E}\left[\left\{\int_{0}^{T}-\mathrm{i} u Y_{\varepsilon}(t) d t\right\} \mathrm{e}^{-\mathrm{i} u \cdot \int_{0}^{T} X_{\varepsilon}^{x}(t) d t}\right] d u
\end{aligned}
$$

Proof. First, by dominated convergence, we can move the gradient inside the integral and inside the expectation operator on the right-hand side in (3.17). Next, differentiating, we obtain straightforwardly the results since $Y(t)=\nabla X^{x}(t)$. We follow the same argument for $X_{\varepsilon}^{x}(t), \int_{0}^{T} X^{x}(t) d t$, and $\int_{0}^{T} X_{\varepsilon}^{x}(t) d t$.

Finally, we state our result on robustness. The proof follows the same steps of the proof of Proposition 4.6 in Benth, Di Nunno, and Khedher [5].

Proposition 3.8. Let $u \widehat{f}(u) \in L^{1}\left(\mathbb{R}^{d}\right)$. For $0 \leq t \leq T$, it holds that

$$
\lim _{\varepsilon \downarrow 0} \nabla \mathbb{E}\left[f\left(X_{\varepsilon}^{x}(t)\right)\right]=\nabla \mathbb{E}\left[f\left(X^{x}(t)\right)\right]
$$

and

$$
\lim _{\varepsilon \downarrow 0} \nabla \mathbb{E}\left[f\left(\int_{0}^{T} X_{\varepsilon}^{x}(t) d t\right)\right]=\nabla \mathbb{E}\left[f\left(\int_{0}^{T} X^{x}(t) d t\right)\right]
$$

\section{Applichtion to Stochastic volatility Models}

Stochastic volatility models describe the joint evolution of the underlying asset price and its variance. Let us first consider the following general stochastic volatility model.

$$
\left\{\begin{array}{l}
d X(t)=\mu X(t-) d t+u(Y(t-)) X(t-) d W^{(1)}(t-)+\int_{\mathbb{R}_{0}}\left(e^{z}-1\right) X(t-) \tilde{N}(d t, d z), \\
d Y(t)=b(t-, Y(t-)) d t+v(t-, Y(t-)) d W^{(2)}(t)+\int_{\mathbb{R}_{0}} \beta(z) \tilde{N}(d t, d z),
\end{array}\right.
$$

Here $X(0)=x, Y(0)>0, \mu \in \mathbb{R}, b$ and $v$ are Lipschitz continuous and differentiable functions on $[0, T] \times \mathbb{R}, u$ is a nonnegative function Lipschitz continuous and differentiable on $\mathbb{R}, \beta$ is a function on $\mathbb{R}, \widetilde{N}$ is a compound Poisson process, and $W^{(1)}$ and $W^{(2)}$ are two correlated standard Brownian motions. We have

$$
d W^{(1)}(t) d W^{(2)}(t)=\rho d t, \quad \rho \in(-1,1) .
$$


Therefore it exists a Brownian motion $\widetilde{W}$, independent of $W^{(1)}$ and $W^{(2)}$ such that we can express $W^{(1)}$ in terms of $\widetilde{W}$ and $W^{(2)}$ as follows

$$
W^{(1)}(t)=\rho W^{(2)}(t)+\sqrt{1-\rho^{2}} \widetilde{W}(t) .
$$

The process $X$ plays the role of the stock price process, while $u(Y)$ is the volatility process. Introduce the following stochastic differential equation

$$
\begin{aligned}
d X^{c, Y}(t) & =\left(\mu+\int_{\mathbb{R}_{0}}\left(1+z-e^{z}\right) \ell(d z)\right) X^{c, Y}(t) d t+u(Y(t)) X^{c, Y}(t) \rho d W^{(2)}(t) \\
& +u(Y(t)) X^{c, Y}(t) \sqrt{1-\rho^{2}} d \widetilde{W}(t), \\
X^{c, Y}(0) & =x .
\end{aligned}
$$

We denote by $V^{Y}=\frac{\partial X^{c, Y}(t)}{\partial x}$. Then we have the following proposition.

Proposition 4.1. Consider the general stochastic volatility model (4.1). Then for $a \in \Gamma$ and $f \in L^{2}(\Omega)$, we have

$$
\begin{aligned}
\Delta & =E\left[f ( X ( T ) , Y ( T ) ) \left(\int_{0}^{T} \frac{a(t) V^{Y}(t)}{u(Y(t)) X^{c, Y}(t)\left(1-\rho^{2}\right)} d W^{(1)}(t)\right.\right. \\
& \left.\left.-\int_{0}^{T} \frac{\rho a(t) V^{Y}(t)}{u(Y(t)) X^{c, Y}(t)\left(1-\rho^{2}\right)} d W^{(2)}(t)\right)\right] .
\end{aligned}
$$

Proof. We denote by $D^{\widetilde{W}}$, the Malliavin derivative with respect to the Brownian motion $\widetilde{W}$. Thus, by Thm.2.2.1 in Nualart [26], we have

$$
\begin{aligned}
D_{t}^{\widetilde{W}} X^{c, Y}(T) & =u(Y(t)) X^{c, Y}(t) \sqrt{1-\rho^{2}}+\int_{t}^{T}\left(\mu+\int_{\mathbb{R}_{0}}\left(1+z-e^{z}\right) \ell(d z)\right) D_{t}^{\widetilde{W}} X^{c, Y}(s) d s \\
& +\int_{t}^{T} D_{t}^{\widetilde{W}}\left(u(Y(s)) X^{c, Y}(s) \rho\right) d W^{(2)}(s) \\
& +\int_{t}^{T} D_{t}^{\widetilde{W}}\left(u(Y(s)) X^{c, Y}(s) \sqrt{1-\rho^{2}}\right) d \widetilde{W}(s) .
\end{aligned}
$$

As the process $Y$ depends only on the Brownian motion $W^{(2)}$ and a jump part, then we have

$$
\begin{aligned}
D_{t}^{\widetilde{W}} X^{c, Y}(T) & =u(Y(t)) X^{c, Y}(t) \sqrt{1-\rho^{2}}+\int_{t}^{T}\left(\mu+\int_{\mathbb{R}_{0}}\left(1+z-e^{z}\right) \ell(d z)\right) D_{t}^{\widetilde{W}} X^{c, Y}(s) d s \\
& +\int_{t}^{T} u(Y(s)) \rho D_{t}^{\widetilde{W}} X^{c, Y}(s) d W^{(2)}(s) \\
& +\int_{t}^{T} u(Y(s)) \sqrt{1-\rho^{2}} D_{t}^{\widetilde{W}} X^{c, Y}(s) d \widetilde{W}(s) .
\end{aligned}
$$


Therefore $D_{t}^{\widetilde{W}} X^{c, Y}(T)=V^{Y}(T)\left(V^{Y}(t)\right)^{-1}\left(u(Y(t)) X^{c, Y}(t) \sqrt{1-\rho^{2}}\right)$. However, the delta is given by

$$
\begin{aligned}
\Delta & =\frac{\partial}{\partial x} \mathbb{E}[f(X(T), Y(T))]=\mathbb{E}\left[f^{\prime}(X(T), Y(T)) \frac{\partial X(T)}{\partial x}\right] \\
& =\mathbb{E}\left[f^{\prime}(X(T), Y(T)) \frac{\partial X(T)}{\partial X^{c, Y}(T)} \frac{\partial X^{c, Y}}{\partial x}\right] .
\end{aligned}
$$

The process $X$ can be written as $X(t)=e^{\widetilde{Z}(t)} X^{c, Y}(t)$, where $\widetilde{Z}(t)=\int_{\mathbb{R}_{0}} z \widetilde{N}(d t, d z)$. Therefore, by the chain rule (Corollary 3.6 in Solé, Utzet, and Vives [32]), we have

$$
D_{t, 0} X(T)=\frac{\partial X(T)}{\partial X^{c, Y}} D_{t}^{\widetilde{W}} X^{c, Y}(T) .
$$

We replace $D_{t}^{\widetilde{W}} X^{c, Y}(T)$ by its expression, we get

$$
D_{t, 0} X(T)=\frac{\partial X(T)}{\partial X^{c, Y}} V^{Y}(T)\left(V^{Y}(t)\right)^{-1}\left(u(Y(t)) X^{c, Y}(t) \sqrt{1-\rho^{2}}\right) .
$$

Hence

$$
\frac{\partial X(T)}{\partial X^{c, Y}(T)} V^{Y}(T)=D_{t, 0} X(T) V^{Y}(t)\left(u(Y(t)) X^{c, Y}(t) \sqrt{1-\rho^{2}}\right)^{-1} .
$$

Therefore, we get the expression for the delta as follows

$$
\Delta=E\left[f(X(T), Y(T)) \int_{0}^{T} a(t) V^{Y}(t)\left(u(Y(t)) X^{c, Y}(t) \sqrt{1-\rho^{2}}\right)^{-1} d \widetilde{W}(t)\right]
$$

where $a(t) \in \Gamma$.

As for the robustness, we can approximate the stochastic volatility model (4.1) by the following

$$
\left\{\begin{aligned}
d X_{\varepsilon}(t) & =\mu X_{\varepsilon}(t-) d t+u\left(Y_{\varepsilon}(t-)\right) X_{\varepsilon}(t-) d W^{(1)}(t-) \\
& +\left(\int_{|z|<\varepsilon}\left(e^{z}-1\right)^{2} \ell(d z)\right)^{\frac{1}{2}} X_{\varepsilon}(t-) d B^{(1)}(t)+\int_{|z| \geq \varepsilon}\left(e^{z}-1\right) X_{\varepsilon}(t-) \tilde{N}(d t, d z), \\
d Y_{\varepsilon}(t) & =b\left(t-, Y_{\varepsilon}(t-)\right) d t+v\left(t-, Y_{\varepsilon}(t-)\right) d W^{(2)}(t) \\
& +\left(\int_{|z|<\varepsilon} \beta^{2}(z) \ell(d z)\right)^{\frac{1}{2}} d B^{(2)}(t)+\int_{|z| \geq \varepsilon} \beta(z) \tilde{N}(d t, d z),
\end{aligned}\right.
$$

where $\left(B^{(1)}, B^{(2)}\right)$ is a Brownian motion independent of $\left(W^{(1)}, W^{(2)}\right)$. By Proposition 3.2, we have the convergence when $\varepsilon$ goes to 0 of the equation (4.4) to the equation (4.1) in $L^{2}(\Omega)$. The convergence of the option price and its delta when $\varepsilon$ goes to 0 follows from Corollary 3.4 and Proposition 3.8.

As an example, we give a slight generalization of the Heston model (see Heston [22]). That is we consider a Heston model with jumps in the underlying asset price. 
Heston model. The Heston model is given by

$$
\begin{aligned}
d X(t) & =r X(t-) d t+\sqrt{Y(t)} X(t-) d W^{(1)}(t) \\
& +\int_{\mathbb{R}_{0}}\left(e^{z}-1\right) X(t-) \tilde{N}(d t, d z), \quad X(0)=x_{1}, \\
d Y(t) & =k(\theta-Y(t)) d t+\eta \sqrt{Y(t)} d W^{(2)}(t), \quad Y(0)>0 .
\end{aligned}
$$

$r$ is a deterministic risk free interest rate, $\theta$ is a long-term variance, $k$ is a mean-reverting rate, and $\eta$ is referred to the volatility of the variance. We assume that $2 k \theta \geq \eta$. The volatility in this model is the square root of the mean reverting process $Y$, introduced by Cox, Ingersoll, and Ross [10]. The square root function is neither differentiable in zero nor globally Lipschitz. In a paper by Alos and Ewald [2], the uniqueness and existence of solution is proved. Moreover, it is proved that $\sqrt{Y(t)}$ is Malliavin differentiable (Corollary 4.2 in Alos and Ewald [2]). We consider the process $X^{c, Y}$ given by

$$
\begin{aligned}
X^{c, Y} & =\left(r+\int_{\mathbb{R}_{0}}\left(1+z-e^{z}\right) \ell(d z)\right) X^{c, Y}(t) d t+\sqrt{Y(t)} \rho X^{c, Y}(t) d W^{(2)}(t) \\
& +\sqrt{Y(t)} X^{c, Y}(t) \sqrt{1-\rho^{2}} d \widetilde{W}(t) .
\end{aligned}
$$

This process is Malliavin differentiable with respect to the Brownian motion $\widetilde{W}$ therefore Proposition 4.1 still applies and taking $u(Y(t))=\sqrt{Y(t)}, V^{Y}(t)=\frac{1}{x} X^{c, Y}(t)$, and $a(t)=\frac{1}{T}$, the delta is given by

$$
\Delta=E\left[f(X(T), Y(T)) \frac{1}{x T}\left(\int_{0}^{T} \frac{d W^{(1)}(t)}{\sqrt{Y(t)}\left(1-\rho^{2}\right)}-\frac{\rho}{1-\rho^{2}} \int_{0}^{T} \frac{d W^{(2)}(t)}{\sqrt{Y(t)}}\right)\right] .
$$

A second example is the Heston model with jumps in the volatility (see Matytsin [25] and Sepp [30]).

Heston model with jumps in the volatility. We consider the following stochastic differential equation

$$
\begin{aligned}
d X(t) & =r X(t-) d t+\sqrt{Y(t)} X(t-) d W^{(1)}(t) \\
& +(\alpha-1) X(t-)(d N(t)-\lambda d t), \quad X(0)=x_{1}, \\
d Y(t) & =k(\theta-Y(t-)) d t+\eta \sqrt{Y(t-)} d W^{(2)}(t)+\beta d J(t), \quad Y(0)=x_{2},
\end{aligned}
$$

where $N$ is a Poisson process with constant intensity $\lambda$ and $J$ is a Poisson process independent of $N$. $\beta$ is a constant. We assume that $2 k \theta \geq \eta$. We consider $\widehat{X}(t)=\alpha^{N(t)} X^{c, Y}(t)$, where

$X^{c, Y}(t)=(\lambda(1-\alpha)+r) X^{c, Y}(t) d t+\sqrt{Y(t)} X^{c, Y}(t) \rho d W^{(2)}(t)+\sqrt{Y(t)} X^{c, Y}(t) \sqrt{1-\rho^{2}} d \widetilde{W}(t)$.

Applying the Itô formula to $\widehat{X}$, we have $\widehat{X}=X$, a.s. By Corollary 4.2 in Alos and Ewald [2] and Theorem 2.2 in Nualart [26], the process $X^{c, Y}$ is Malliavin differentiable with respect to the Brownian motion $\widetilde{W}$. Therefore applying Proposition 4.1, with $u(Y(t))=\sqrt{Y(t)}$, 
$V^{Y}(t)=\frac{1}{x} X^{c, Y}(t)$, and $a(t)=\frac{1}{T}$, the delta is given by

$$
\Delta=E\left[f(X(T), Y(T)) \frac{1}{x T}\left(\int_{0}^{T} \frac{d W^{(1)}(t)}{\sqrt{Y(t)}\left(1-\rho^{2}\right)}-\frac{\rho}{1-\rho^{2}} \int_{0}^{T} \frac{d W^{(2)}(t)}{\sqrt{Y(t)}}\right)\right] .
$$

4.1. Stability of option prices (the BNS model). We consider the following BNS model,

$$
\left\{\begin{array}{l}
d X(t)=(\mu+\beta Y(t)) d t+\sqrt{Y}(t) d W(t)+\rho d Z(t), \quad X(0)=x \\
d Y(t)=-\lambda Y(t) d t+d Z(t), \quad Y(0)>0
\end{array}\right.
$$

where the parameters $\mu, \beta, \rho$, and $\lambda$ are real constants with $\lambda>0$ and $\rho \leq 0 . Z=Z(t)$, $0 \leq t \leq T$ is a subordinator (i.e. increasing Lévy process). We assume that $Z$ has no deterministic drift and its Lévy measure has density $\omega(z)$, so that the cumulant transform $k(\theta)=\log \mathbb{E}\left[e^{\theta Z_{1}}\right]$, where it exists takes the form

$$
k(\theta)=\int_{\mathbb{R}_{+}}\left(e^{\theta z}-1\right) \omega(z) d z .
$$

We denote by $N$ the random measure associated with the jumps of $Z$. We consider a parameter $\lambda_{\varepsilon}, 0<\varepsilon<1$, such that

$$
\lim _{\varepsilon \rightarrow 0} \lambda_{\varepsilon}=\lambda \text {. }
$$

Notice that in this case by triangular inequality we have

$$
\left|\lambda_{\varepsilon}\right| \leq|\lambda|+\left|\lambda_{\varepsilon}-\lambda\right| \text {. }
$$

In particular when $\varepsilon$ is sufficiently small, we have $\left|\lambda_{\varepsilon}-\lambda\right| \leq 1$. Therefore $\left|\lambda_{\varepsilon}\right| \leq a$, where $a=1+|\lambda|$. Therefore, we have the following approximation for the BNS model

$$
\left\{\begin{aligned}
d X_{\varepsilon}(t) & =\left(\mu+\beta Y_{\varepsilon}(t)\right) d t+\sqrt{Y_{\varepsilon}(t)} d W(t)+\rho d Z(t), \quad X_{\varepsilon}(0)=x \\
d Y_{\varepsilon}(t) & =-\lambda_{\varepsilon} Y_{\varepsilon}(t) d t+d Z(t), \quad Y_{\varepsilon}(0)>0 .
\end{aligned}\right.
$$

In the following, we study the robustness of the BN-S model and the associated option price. The computation of the delta is studied in Benth, Groth, and Wallin [3].

Lemma 4.2. The system given by (4.6) converges to (4.5) almost surely when $\varepsilon$ goes to 0.

Proof. The process $Y_{\varepsilon}$ is given by

$$
Y_{\varepsilon}(t)=e^{-\lambda_{\varepsilon} t} Z(0)+\int_{0}^{t} e^{\lambda_{\varepsilon}(s-t)} d Z(s)
$$

As $e^{\lambda_{\varepsilon} s} \leq e^{a T}$, then by dominated convergence theorem, we can take the limit inside the integral in (4.7) and we have the almost sure convergence of the process $Y_{\varepsilon}$ to the process $Y$ when $\varepsilon$ goes to 0 . The process $X_{\varepsilon}$ is given by

$$
X_{\varepsilon}(t)=x+\int_{0}^{t}\left(\mu+\beta Y_{\varepsilon}(s)\right) d s+\int_{0}^{t} \sqrt{Y_{\varepsilon}}(t) d W(t)+\rho Z(t) .
$$

As we have $\left|Y_{\varepsilon}\right| \leq Z(0)+e^{a T} Z(T)$, then by dominated convergence theorem, we can take the limit inside the integral in (4.8) and the result follows. 
We consider a European option written on $S(t)=e^{X(t)}, 0 \leq t \leq T$, with exercise time $T$ and payoff function $f: \mathbb{R} \rightarrow \mathbb{R}$. The arbitrage free price is given by

$$
C(t)=e^{-r(T-t)} \mathbb{E}^{Q}[f(S(T) \mid \mathcal{F}(t)],
$$

where the parameter $r$ is the risk free instantaneous interest rate of a bond used as a numéraire and the measure $Q$ is an equivalent martingale measure (i.e, it is a measure equivalent to $\mathbb{P}$ and under which the discounted price process $e^{-r t} S(t)$ is a martingale). In our case, the market is incomplete and there will be an infinity of equivalent martingale measures ( denoted EMM's). Among the wide class of the EMM's, Nicolato and Venardos [27] studied a structure preserving subclass, a subclass under which the log price process and its volatility are again described by a model of the type (4.5). In our setting, we will deal with this structure preserving subclass.

We denote by $\mathcal{M}$ the subset of EMM's such that the log-price process $X_{\varepsilon}$ is still described by a $\mathrm{BN}-\mathrm{S}$ model. Introduce the following class

$$
\mathcal{Y}=\left\{y: \mathbb{R}+\rightarrow \mathbb{R}_{+} \mid \int_{\mathbb{R}+}(\sqrt{y(z)}-1)^{2} \omega(z) d z<\infty\right\}
$$

and for $y \in \mathcal{Y}$, we set

$$
\omega^{y}(z)=y(z) \omega(z)
$$

Since $\int_{|z| \leq 1} z \omega^{y}(z) d z<\infty$, we can also define

$$
k^{y}(\theta)=\int_{\mathbb{R}_{+}}\left(e^{\theta z}-1\right) \omega^{y}(z) d z, \quad \text { for } \quad \operatorname{Re}(\theta)<0 .
$$

The following theorem is due to Nicolato and Venardos [27].

Theorem 4.3. Let $y \in \mathcal{Y}$. Then the processes

$$
\psi(t)=\sqrt{Y(t)}^{-1}\left(r-\mu-\left(\beta+\frac{1}{2}\right) Y(t)-k^{y}(\rho)\right)
$$

and

$$
\psi_{\varepsilon}(t)={\sqrt{Y_{\varepsilon}(t)}}^{-1}\left(r-\mu-\left(\beta+\frac{1}{2}\right) Y_{\varepsilon}(t)-k^{y}(\rho)\right),
$$

where $k^{y}$ is given by (4.10), are such that

$$
P\left(\int_{0}^{T} \psi^{2}(s) d s<\infty\right)=1
$$

and

$$
P\left(\int_{0}^{T} \psi_{\varepsilon}^{2}(s) d s<\infty\right)=1 .
$$

The processes

$$
L^{y}(t)=\exp \left\{\int_{0}^{t} \psi(s) d W(s)-\frac{1}{2} \int_{0}^{t} \psi^{2}(s) d s+\int_{0}^{t} \int_{0}^{\infty} \log (y(s, z)) N(d s, d z)\right.
$$




$$
\left.+\int_{0}^{t} \int_{0}^{\infty}(1-y(s, z)) \omega(z) d z d s\right\}, \quad 0 \leq t \leq T
$$

and

$$
\begin{aligned}
L_{\varepsilon}^{y}(t) & =\exp \left\{\int_{0}^{t} \psi_{\varepsilon}(s) d W(s)-\frac{1}{2} \int_{0}^{t} \psi_{\varepsilon}^{2}(s) d s+\int_{0}^{t} \int_{0}^{\infty} \log (y(s, z)) N(d s, d z)\right. \\
& \left.+\int_{0}^{t} \int_{0}^{\infty}(1-y(s, z)) \omega(z) d z d s\right\}, \quad 0 \leq t \leq T .
\end{aligned}
$$

are density processes. The probability measures defined by

$$
d Q_{\varepsilon}^{y}=L(T) d \mathbb{P}
$$

and

$$
d Q_{\varepsilon}^{y}=L_{\varepsilon}(T) d \mathbb{P}
$$

are EMM and the dynamic of $X$ under $Q^{y}$ is given by

$$
\left\{\begin{array}{l}
d X(t)=\left(r-k^{y}(\rho)-\frac{1}{2} Y(t)\right) d t+\sqrt{Y}(t) d W^{y}(t)+\rho d Z(t) \\
d Y(t)=-\lambda Y(t) d t+d Z(t), \quad Y(0)>0
\end{array}\right.
$$

where $W^{y}(t)=W(t)-\int_{0}^{t} \psi(s) d s$ is a $Q^{y}$-Brownian motion and $Z(t)$ is a $Q^{y}$-Lévy process. The processes $W^{y}$ and $Z$ are independent under $Q^{y}$. The dynamic of $X_{\varepsilon}$ under $Q_{\varepsilon}^{y}$ is given by

$$
\left\{\begin{aligned}
d X_{\varepsilon}(t) & =\left(r-k^{y}(\rho)-\frac{1}{2} Y_{\varepsilon}(t)\right) d t+\sqrt{Y}_{\varepsilon}(t) d W_{\varepsilon}^{y}(t)+\rho d Z(t) \\
d Y_{\varepsilon}(t) & =-\lambda_{\varepsilon} Y_{\varepsilon}(t) d t+d Z(t), \quad Y_{\varepsilon}(0)>0,
\end{aligned}\right.
$$

where $W_{\varepsilon}^{y}(t)=W(t)-\int_{0}^{t} \psi_{\varepsilon}(s) d s$ is a $Q_{\varepsilon}^{y}$-Brownian motion, and $Z(t)$ is a $Q_{\varepsilon}^{y}$-Lévy process. $Z_{1}$ has Lévy density $\omega^{y}(z)$ and cumulant transform $k^{y}(\theta)$ respectively given by (4.9) and (4.10) and the processes $W_{\varepsilon}^{y}$ and $Z$ are independent under $Q_{\varepsilon}^{y}$. Hence $Q^{y}, Q_{\varepsilon}^{y} \in \mathcal{M}$.

In the following lemma, we study the robustness of the dynamic of $X$ under the new measure $Q^{y}$.

Lemma 4.4. The system of equation (4.12) converges to (4.11) almost surely when $\varepsilon$ goes to 0 .

Proof. For $0<\lambda_{\varepsilon}<a$, we have $\left|Y_{\varepsilon}(t)\right| \geq e^{-a t}(Z(0)+Z(t))$. Therefore $\left|\frac{1}{\sqrt{Y_{\varepsilon}}}\right| \leq K(t, \omega)$, where $K(t, \omega)=\frac{1}{e^{-a t}(Z(0)+Z(t))}$. As $\left|\frac{1}{\sqrt{Y_{\varepsilon}}}\right|$ is defined and continuous for $Y_{\varepsilon}>0$, then $\frac{1}{\sqrt{Y_{\varepsilon}}}$ converges to $\frac{1}{\sqrt{Y}}$ almost surely when $\varepsilon$ goes to 0 . Therefore $\psi_{\varepsilon}$ converges to $\psi$ when $\varepsilon$ goes to 0 and $\left|\psi_{\varepsilon}\right| \leq C(t, \omega)$, where $C$ is a constant depending on time $t$. By dominated convergence, taking the limit inside the integral in the following expression $W_{\varepsilon}^{y}(t)=W(t)-$ $\int_{0}^{t} \psi_{\varepsilon}(s) d s$, we have the convergence of $W_{\varepsilon}^{y}(t)$ to $W^{y}(t)$. Then following the steps of the proof of Lemma 4.2, we get the result. 
In the following, we study the convergence of the option price under the risk-neutral equivalent martingale measure $Q^{y}$. In this direction, we mention a paper by Benth, Di Nunno and Khedher [6] in which they study the robustness of option prices after a change of measure in markets driven by jump-diffusions. Consider the price process $S_{\varepsilon}(t)=e^{X_{\varepsilon}(t)}$, $0 \leq t \leq T$. Let $\mathcal{F}_{\varepsilon}(t)$ be the filtration generated by the Brownian motion $W_{\varepsilon}^{y}(t)$ and the Lévy process $Z(t)$. The option price is given by

$$
C_{\varepsilon}(t)=e^{-r(T-t)} \mathbb{E}^{Q_{\varepsilon}}\left[f\left(S_{\varepsilon}(T) \mid \mathcal{F}_{\varepsilon}(t)\right] .\right.
$$

To evaluate the latter expression, we use a Fourier transform approach which extend the method considered in the paper by Nicolato and Venardos [27]. In their approach, they have some restrictions on the Lévy measure which we don't need to consider. We first, state the following results.

The integrated variance over the time period $[t, T]$, is given by $\sigma_{\varepsilon}^{* 2}(t, T)=\int_{t}^{T} Y_{\varepsilon}(s) d s$ and a simple computation shows that

$$
\sigma_{\varepsilon}^{* 2}(t, T)=\lambda_{\varepsilon}^{-1}\left(1-e^{-\lambda_{\varepsilon}(T-t)}\right) Y_{\varepsilon}(t)+\int_{t}^{T} \lambda_{\varepsilon}^{-1}\left(1-e^{-\lambda_{\varepsilon}(T-s)}\right) d Z(s) .
$$

Using the Key formula in Eberlein and Raible [19], the Fourier transform of the conditional integrated variance $\sigma_{\varepsilon}^{* 2}(t, T)$ is computed as

$$
\mathbb{E}^{Q_{\varepsilon}}\left[\exp \left\{-i u \sigma_{\varepsilon}^{* 2}(t, T)\right\} \mid \mathcal{F}_{\varepsilon}(t)\right]=\exp \left\{-i u Y_{\varepsilon}(t) \epsilon_{\varepsilon}(t, T)+\int_{t}^{T} k\left(-i u \epsilon_{\varepsilon}(s, T)\right) d s\right\},
$$

where $\epsilon_{\varepsilon}(s, T)=\lambda_{\varepsilon}^{-1}\left(1-e^{-\lambda_{\varepsilon}(T-s)}\right)$. Due to the Theorem 2.2 in Nicolato and Venardos [27], the Fourier transform of the $\log$-price $X_{\varepsilon}$ given the information up to the time $t \leq T$ is given by

$$
\begin{aligned}
\phi_{\varepsilon}(u) & =\mathbb{E}^{Q_{\varepsilon}}\left[\exp \left\{-i u X_{\varepsilon}(T)\right\} \mid \mathcal{F}_{\varepsilon}(t)\right] \\
& =\exp \left\{-i u\left(X_{\varepsilon}(t)+r(T-t)-k^{y}(\rho)(T-t)\right)+\frac{1}{2}\left(-i u-u^{2}\right) Y_{\varepsilon}(t) \epsilon_{\varepsilon}(t, T)\right. \\
& \left.+\int_{t}^{T} k\left(h_{\varepsilon}(s, z)\right) d s\right\},
\end{aligned}
$$

where $h_{\varepsilon}(s, z)=-i u \rho+\frac{1}{2}\left(-i u-u^{2}\right) \epsilon_{\varepsilon}(s, T)$. Notice that, for all $u \in \mathbb{R}, \phi_{\varepsilon}(u)$ converges to $\phi(u)$ when $\varepsilon$ goes to 0 .

Assume $f, \widehat{f} \in L^{1}(\mathbb{R})$. Then the option price is given by

$$
\begin{aligned}
C_{\varepsilon}(t) & =e^{-r(T-t)} \mathbb{E}^{Q_{\varepsilon}}\left[f\left(X_{\varepsilon}(T) \mid \mathcal{F}_{\varepsilon}(t)\right]\right. \\
& =e^{-r(T-t)} \frac{1}{2 \pi} \mathbb{E}^{Q_{\varepsilon}}\left[\int_{\mathbb{R}} e^{-i u X_{\varepsilon}(T)} \widehat{f}(y) d y \mid \mathcal{F}_{\varepsilon}(t)\right] \\
& =e^{-r(T-t)} \frac{1}{2 \pi} \int_{\mathbb{R}} \widehat{f}(y) \mathbb{E}^{Q_{\varepsilon}}\left[e^{-i u X_{\varepsilon}(T)} \mid \mathcal{F}_{\varepsilon}(t)\right] d y \\
& =e^{-r(T-t)} \frac{1}{2 \pi} \int_{\mathbb{R}} \widehat{f}(y) \phi_{\varepsilon}(y) d y .
\end{aligned}
$$


Lemma 4.5. The option price $C_{\varepsilon}(t)$ converges to $C(t)$ when $\varepsilon$ goes to 0 .

Proof. From Jensen inequality for the conditional expectation, we have

$$
\left|\phi_{\varepsilon}(y)\right|=\left|\mathbb{E}^{Q_{\varepsilon}}\left[\exp \left\{-i u X_{\varepsilon}(T)\right\} \mid \mathcal{F}_{\varepsilon}(t)\right]\right| \leq \mathbb{E}^{Q_{\varepsilon}}\left[\left|\exp \left\{-i u X_{\varepsilon}(T)\right\}\right| \mid \mathcal{F}_{\varepsilon}(t)\right] \leq 1
$$

Therefore by dominated convergence, we can take the limit inside the integral in (4.13) and the result follows.

\section{REFERENCES}

[1] Asmussen, S., and Rosinski, J. (2001). Approximations of small jump Lévy processes with a view towards simulation. J. Appl. Prob., 38, pp. 482-493.

[2] Alos, E., and Ewald, C.O. (2007). Malliavin differentiability of the Heston volatility and applications to option pricing. E-print, MPRA Paper 3237, University Library of Munich, Germany.

[3] Benth, F. E., Groth, M., and Wallin. O. (2010). Derivative-free Greeks for the Barndorff-Nielsen and Shephard stochastic volatility model, Stochastics, 82(3), pp. 291-313.

[4] Benth, F. E., Di Nunno, G., and Khedher, A. (2009). Lévy models robustness and sensitivity. In Volume XXV of the series QP-PQ, Quantum Probability and White Noise Analysis, H. Ouerdiane and A. Barhoumi (eds.), World Scientific, pp. 153-184.

[5] Benth, F. E., Di Nunno, G., and Khedher, A. (2010). Robustness of option prices and their deltas in markets modelled by jump-diffusions. E-print, No. 2, January, Department of Mathematics, University of Oslo, Norway. To appear in Comm. Stoch. Analysis.

[6] Benth, F. E., Di Nunno, G., and Khedher, A. (2010). A note on convergence of option prices and their Greeks for Lévy models. E-print, No. 18, November, Department of Mathematics, University of Oslo, Norway.

[7] Barndorff-Nielsen, O.E., Shephard, N. (2001). Non-Gaussian Ornstein-Uhlenbeck-based models and some of their uses in financial economics, J. R. Stat. Soc. Ser. B Stat. Methodol., 63(2), pp. $167-241$.

[8] Caramellino, L., and Marchisio, V. (2010). Malliavin Greeks for Complex Asian options in a jump diffusion setting. Preprint.

[9] Carr, P., and Madan, D. B. (1998). Option valuation using fast Fourier transform. J. Comp. Finance, 2, pp. $61-73$.

[10] Cox, J. C., Ingersoll, J. E., and Ross, S. A. (1985). A theory of the term structure of interest rates. Econometrica, 53, pp. 385-408.

[11] Cont, R., and Tankov, P. (2004). Financial Modelling with Jump Processes. Chapman Hall.

[12] Cont, R., Tankov, P., Voltchkova, E. (2007). Hedging with options in models with jumps. appeared in Stochastic Analysis and Applications - the Abel Symposium 2005, Springer.

[13] Cass, T. R., and Friz, P. K. (2007). The Bismut-Elworthy-Li formula for jump-diffusions and applications to Monte Carlo methods in finance. arXiv:math/0604311.

[14] Davis, M. H. A., and Johansson, M. P.(2006). Malliavin Monte Carlo Greeks for jump-diffusions. Stoch. Processes. Appl., 116(1), pp. 101-129.

[15] Di Nunno, G. (2008). On orthogonal polynomials and the Malliavin derivative for Lévy stochastic measures. SMF Séminaires et Congrès, 16, pp. 55-70.

[16] Di Nunno, G., Øksendal, B., and Proske, F. (2004). White noise analysis for L'evy processes. Journal of Functional Analysis, 206, pp. 109-148.

[17] Di Nunno, G. and Rozanov, Yu.A. (2007). Stochastic integrals and adjoint derivatives. In Stochastic Analysis and its Applications, Springer.

[18] El-Khatib, Y., and Privault, N. (2004). Computation of Greeks in a market with jumps via the Malliavin calculus. Finance and Stochastics, 8, pp. 161-179.

[19] Eberlein, E. and S. Raible (1999). Term structure models driven by general Lévy processes. Mathematical Finance 9, pp. 31-53. 
[20] Folland, G. B. (1984). Real Analysis - Modern Techniques and their Applications. John Wiley \& Sons.

[21] Fournié, E., Lasry, J. M., Lébucheux, J., Lions, P. L., and Touzi, N. (1999). Applications of Malliavin calculus to Monte Carlo methods in finance, Finance Stoch., 3, pp 391-412.

[22] Heston, H. (1993). A closed-form solution for options with stochastic volatility with applications to bond and currency options, Review of Financial Studies, 6, pp 327-343.

[23] Ikeda, N. and Watanabe, S. (1981). Stochastic Differential Equations and Diffusion Processes. Elsevier Science.

[24] Karatzas, I., and Shreve, S. E. (1991). Brownian Motion and Stochastic Calculus. Springer.

[25] Matytsin, A.(1999). Modeling volatility and volatility derivatives. Columbia Practitioners Conference on the Mathematics of Finance, Presentation.

[26] Nualart, D. (1995). The Malliavin calculus and related topics. Springer.

[27] Nicolato, E. and Venardos, E (2003). Option pricing in stochastic volatility models of the OrnsteinUhlenbeck type, Mathematical Finance., 13, pp. 445-466.

[28] Protter, P. (2005) Stochastic Integration and Differential Equations. Springer.

[29] Rydberg, T. H. (1997). The normal inverse Gaussian Lévy process: simulation and approximation, Comm. Stat. Stoch. Models, 13, pp. 887-910.

[30] Sato, K.I. (1999). Lévy Processes and Infinitely Divisible Distributions. Cambridge University Press.

[31] Sepp, A. (2008). Pricing options on realized variance in the Heston model with jumps in returns and volatility, Journal of Computational Finance, 11, pp. 33-70.

[32] Solé, J. L., Utzet, F., and Vives, J.(2007). Canonical Lévy process and Malliavin calculus. Stochastic processes and their Applications., 117, pp 165-187.

A. Khedher: Center of Mathematics for Applications, University of Oslo, PO Box 1053 Blindern, N-0316 OsLo, NorWAY

E-mail address: asma.khedher@cma.uio.no 